\title{
File Sharing between Peer-to-Peer using Network Coding Algorithm
}

\author{
Rathod Vijay U. \\ PG Student, \\ MBES College Of Engineering, Ambajogai
}

\author{
V.R. Chirchi \\ PG Dept, \\ MBES College Of Engineering, Ambajogai
}

\begin{abstract}
Network coding is a good improvement of network routing to improve network throughput and provide high reliability. It allows a node to generate output messages by encoding its received messages. Peer-to-peer networks are a perfect place to apply network coding due to two reasons: 1 . in peer-to-peer network, the topology is not fixed. So, it is very much easier to create the topology which suits the network coding; 2 . Peer-to-peer network every nodes are end hosts, so it is easier to perform the complex operation related to network coding like decoding and encoding rather than storing and forwarding the message. In this paper, as propose an algorithm to apply network coding algorithm to peer-to-peer file sharing which employs a peer-to-peer network to distribute files resided in a web server or a file server. The scheme exploits a special type of network topology called combination network. It was proved that combination networks can achieve unbounded network coding gain measured by the ratio of network throughput with network coding to that without network coding. Here network coding algorithm encodes a file into multiple messages and divides peers into multiple groups with each group responsible for relaying one of the messages. The encoding algorithm is designed to satisfy the property that any subset of the messages can be used to decode the original file as long as the size of the subset is sufficiently large. To meet this requirement, here first define an algorithm which satisfies the desired property, and then connect peers in the same group to flood the corresponding message, and connect peers in different groups to distribute messages for decoding. This paper has considered number of theoretical and practical scenarios where network coding or its variant is applied on peer-to-peer file sharing based on Network coding with the aim to improve performance parameters like throughput and reliability. This paper has mainly focused on the comparative analysis of file sharing between peer-to-peer using network coding algorithms.
\end{abstract}

\section{Keywords}

Network Coding Algorithm, Peer-to-Peer Networks, Webbased Applications, File Sharing, Multicast

\section{INTRODUCTION}

In the last decades, the Internet has witnessed great increase of different types of web-based applications, ranging from web-based file sharing to video broadcasting or conferencing. Web-based applications have acquired more and more interests due to the flexibility and easy accessibility. Many such applications involve one source (server) and multiple destinations (receivers). However, due to lack of multicast support over the Internet, these applications usually suffer from the scalability problem, which limits the number of receivers involved. Peer-to-peer is a good technology that can implement multicast at the application layer, where receivers (peers) not only receive data, but also forward data. By incorporating peer-to-peer technology into web-based applications, the scalability problem can be eliminated, i.e., the system performance (throughput, latency, etc.) will not be impaired when there are more users in the system.

In this paper, here consider applying peer-to-peer technology to file sharing services, in which a web server or a file server holds a file that is requested by multiple clients (receivers). In most peer-to-peer network, peers usually are end users' personal computers which may have limited resources (such as bandwidth, CPU time etc) or even be unstable. It is critical for the file sharing system to be reliable and resilient while achieving good throughput at the same time.

Application layer multicast can be incorporated in peer-topeer technology. Scalability issue of web-based applications can be easily eliminated with the help of peer-to-peer networking. Throughput and reliability of a system can be improved by using network coding algorithm. By using network coding algorithm for multicast can fully utilize the network capacity.

Network coding is another good technology that can be employed to improve system throughput and reliability. Here give a brief introduction on network coding. In today's network, messages are generally transferred by routing through intermediate nodes between the source and the destination, i.e., by having intermediate nodes store and forward messages. In fact, routing is not the only operation that can be performed at a node. Recently, network coding has collected as a good improvement of network routing to improve network throughput and provide high reliability. Network coding refers to a scheme where a node is allowed to generate output messages by encoding (i.e., computing certain functions of) it's received messages. Thus, network coding allows information to mix, in contrast to the traditional routing approach where each node simply forwards received messages. Network coding has a wide range of applications from wireless networks [17], [28] to network tomography [18]. Network coding can greatly improve the throughput of a multicast network. Let's first use the well-known butterfly network in Fig. 1 to demonstrate the advantage of network coding for multicast. 


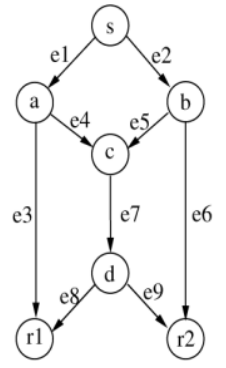

(a)

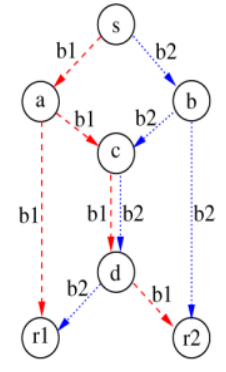

(b)

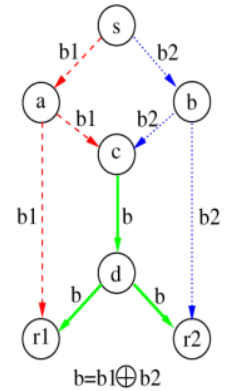

(c)
Fig 1: Illustration of the advantage of network coding for multicast. (a) The butterfly network represented by a

DAG. (b) Multicast without network coding. (c) Multicast with network coding [1].

In the figure, node $\mathrm{s}$ is the source, nodes $\mathrm{r} 1$ and $\mathrm{r} 2$ are two receivers, all edges have capacity 1 , which means that the edge can only transmit 1 unit of data (bit) per unit time (second), and source s has two bits, b1 and b2 to multicast to both $\mathrm{r} 1$ and $\mathrm{r} 2$. First we use the traditional multicast without network coding as shown in Fig. 1(b). Without loss of generality, we use the dashed line (red) to represent bit b1, the dotted line (blue) to represent bit b2 and the bold line (green) to represent both bits b1 and b2. Bit b1 can reach $\mathrm{r} 1$ in two seconds, and bit b2 can reach $\mathrm{r} 2$ in two seconds. When node $\mathrm{c}$ receives both bits, it forwards them in sequence. Suppose it forwards bit b1 first. Then $\mathrm{r} 1$ receives both bits in 4 seconds and $\mathrm{r} 2$ receives both bits in 5 seconds. Now consider using network coding on link $\mathrm{c}-\mathrm{d}$ as shown in Fig. 1(c). When node c receives both bits, it first mixes them by performing an exclusive OR (XOR) operation. Then it sends the mixed bit $b$ to $\mathrm{d}$ node. When nodes $\mathrm{r} 1$ or $\mathrm{r} 2$ receive the mixed bit, it can recover the original bits $\mathrm{b} 1$ and $\mathrm{b} 2$ by XO-Ring the mixed bit and the other received bit. The entire transmission can be completed in 4 seconds. The definition of link $\mathrm{c}$ to $\mathrm{d}$ throughput $(\gamma)$ from node $\mathrm{c}$ to $\mathrm{d}$ is given by

\section{$\gamma=\underline{\text { Number of bits from node } \mathrm{c} \text { to node } \mathrm{d}}$ Observation duration}

Peer-to-peer (overlay) networks are a perfect place to apply network coding algorithm due to two reasons: 1 . in peer-topeer network, the topology is not fixed. So, it is very much easier to create the topology which suits the network coding; 2. Peer-to-peer network every nodes are end hosts, so it is easier to perform the complex operation related to network coding algorithm like decoding and encoding rather than storing and forwarding the message[1].

Network coding can be viewed as a generalized routing scheme and can be applied to any types of routing, i.e. uncast, multicast and broadcast, to enable a more efficient data transmission.

There are two main streams of research in the field of network coding. One stream investigates efficient encoding-anddecoding algorithms to increase the data transmission rate while reducing the computational cost and the other emphasizes the applications of network coding.

For the first stream, regarding the encoding functions to combine the received information at intermediate nodes, there have been a number of encoding-and-decoding algorithms, e.g. linear network coding (Li et al, 2003), algebraic network coding (Koetter and Médard, 2003), convolution network coding (Li and Yeung, 2006) and so on. Among them, one of the most fundamental and widely used coding methods is linear network coding, where packets are linearly combined at intermediate node. Introduced in 2003, linear network coding always suffices to achieve the theoretical maximum throughput for multicast sessions (Li et al, 2003).

This paper is organized as follows: In section II, Literature Review of paper. In section III, Explained Proposed System. In section IV, Advantages of Proposed Algorithm and in section V and VI, future work and conclusion respectively.

\section{LITERATURE REVIEW}

Numbers of network coding scheme have been proposed in past for multicast networks some are explaining as follows:

- 2000: Concept introduced: In a landmark paper, Rudolf Ahlswede, Ning CAI, Shuo-Yen Robert Li and Raymond W. Yeung showed the potential power of network coding in multicast networks where all receivers get identical information. They proved that good (informative) codes exist, although they did not describe a method for designing them [2].

- 2002: Another important performance parameter is the security of the network. CAI and Yeung considered the problem of using network coding to achieve perfect information security against a wire tapper who can eavesdrop on a limited number of network links, and presented the construction of a secure linear network code for this purpose [30].

- 2003: Important steps taken toward practical implementation. Li, Yeung and CAI showed network coding for multicast networks can rely on mathematical functions involving only addition and multiplication, which reduces the complexity of designing codes. And two of us (Koetter and Médard) introduced a powerful algebraic framework for analyzing coding approaches and simplifying code design [3] [4].

- 2005-2006: Valuable design algorithms published. Sidharth Jaggi, then at Caltech, with Peter Sanders of the University of Karlsruhe in Germany, one of us (Effros) and collaborators and, separately, Tracey Ho of Caltech, with the three of us and others, published low-complexity algorithms for designing the functions used by each node in a multicast network. The first paper gave a systematic approach for designing functions; the second showed that choosing functions randomly and independently for each node should work just as well[6][7].

- 2005: One important performance parameter is the network cost incurred for a given set of connections, and the complexity associated with the computation of the sub graphs needed to provide the connections. While the minimum cost multicast problem in routed networks requires the finding of a directed Steiner tree, which is NP-hard, the same problem in coded networks can be solved by a linear program in polynomial time and also be implemented in a decentralized manner [15].

- 2006: Applications for wireless networks explored. D.M.Chiu, R.W.Yeung, J.Huang, and B.Fan, 
demonstrated the potential benefits of network coding for wireless applications and characterized scenarios in which the approach would be particularly helpful [13].

- 2014: It applies linear network coding to peer-topeer file sharing and presents a high performance peer-to-peer file sharing system, PPFEED. Peer-toPeer File Sharing Based on Network Coding (PPFEED) utilizes combination networks as its overlay topology prototype. Combination networks demonstrate great performance gain under linear network coding. It proposes a simple and efficient deterministic linear network coding scheme for combination networks and applies to PPFEED. As a result, PPFEED inherits its high performance when applied network coding and presents its superiority compared to other existing peer-to-peer file sharing systems. They showed that PPFEED can achieve $15 \%-20 \%$ higher throughput than Narada which is an Application lifecycle management (ALM) system without network coding. Besides, it achieves higher reliability and resiliency [1].

\section{PROPOSED SYSTEM}

In past proposed scheme has some problem to remove these problems, in this paper a network coding algorithm for multicast reduces the number of transmission has been proposed. The main aim of this work is to reduce the total number of transmission in multicast network and also to reduce the bandwidth consumption in multicast network using Bit Torrent file sharing protocol, by implementing network coding algorithm. Here abstract diagram of file sharing between peer-to-peer using network coding algorithms as shown in figure below.

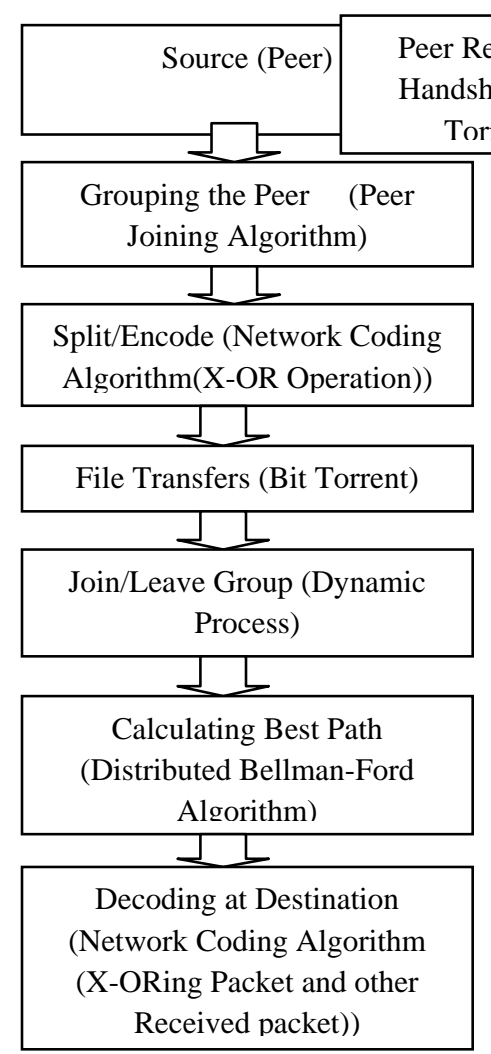

Fig 2: Abstract Diagram of File Sharing Between Peer to Peer Using Network Coding Algorithm

\subsection{Bit Torrent File Sharing Protocol}

Bit torrent is a protocol that enables fast downloading of large files using minimum internet bandwidth. The most popular video, audio or software files can be transferred faster and cheaper by using bit torrent. Bit Torrent is massaging sharing or handshake protocol. Differ from HTTP, bit torrent is rises to full downloading speed very quickly and maintains this speed throughout. Bit torrent has multiple applications such as file sharing, peer-to-peer television, content distribution.

\subsection{Peer Joining}

Here assume that the server is well-known whose IP address is known to all the peers by some address translation service such as DNS (Domain Name System). When a peer wants to retrieve a file hosted by the server, it initiates a join process by sending a JOIN request to the server.

The server maintains various counters and lists. For each group $\mathrm{G}_{\mathrm{i}}$, the server maintains a counter $\mathrm{gc}_{\mathrm{i}}$ to store the number of peers in the group and a list $\mathrm{gl}_{\mathrm{i}}$ to store the list of clusters which include the peers in the group. For each cluster $\mathrm{C}_{\mathrm{i}}$, the server maintains a list $\mathrm{cl}_{\mathrm{i}}$ to store the groups which include peers in the cluster. Besides, the server maintains a list of existing peers and their respective residue upload bandwidths and IP addresses for each group. As more and more peers join the system, it is resource consuming to maintain a full list of peers for each group. The server will keep a partial list of peers with the largest residue upload bandwidths. Meanwhile, peers will report to the server their updated residue upload bandwidths periodically to update the partial list on the server. Although the server is responsible for bootstrapping the peers, it will not be the bottleneck of the system because once each peer receives the list, it communicates with other peers for topology construction and data dissemination. When the server receives a peer's join request, it assigns the peer to a group. Then the server sends the list of peers of that group to the joining peer and updates the number of peers in that group.

The server will assign the new peer to a group based on following factors. First, the peer is assigned to a cluster based on its coordinate. If the number of groups which include peers in the cluster is less than, the new peer will be assigned to one of the groups which include no peers in the cluster. When there are multiple such groups, the group spans the least number of clusters is selected. Tie is broken by picking the group with less number of peers in the group. The rationale behind this is that here want to minimize the logical links between different clusters and ensure that peers can receive sufficient "innovative" messages, i.e., messages from different groups, within the cluster to perform decoding.

Table 1. Peer Joining Algorithm [1]

INPUT : joining peer $\mathrm{v}$
OUTPUT $:$ updated overlay network
BEGIN
//suppose the cluster corresponding to peer $\mathrm{v}$ is $\mathrm{C}_{\mathrm{i}}$
if $\left|\mathrm{cl}_{\mathrm{i}}\right|<\mathbf{k}$
$\mathrm{S}_{\mathrm{i}}=$ the set of groups not in $\mathrm{cl}_{\mathrm{i}}$;
else
$\mathrm{Si}=$ the set of groups in $\mathrm{cl}_{\mathrm{i}}$;
Pick a group $\mathrm{g}_{\mathrm{i}} \mathrm{S}$ such that $\mathrm{gl}_{\mathrm{i}}$ is the smallest;
if multiple groups have the same smallest gl, pick a group gi
with less $\mathrm{gc}_{\mathrm{i}}$;
Peer $\mathrm{v}$ is assigned to group $\mathrm{g}_{\mathrm{i}}$.
END


After receiving the list of peers, the new peer will contact them and create overlay links with them. These peers are called intra-neighbors of the new peer because they are within the same group. In contrast, the neighbors which are in different groups are called inter-neighbors. The new peer asks one of its intra-neighbors to provide a list of its interneighbors. When picking the intra-neighbor, higher priority is given to the peer in the same cluster. The new peer then takes the list of peers as its inter-neighbors.

The topology of the peer-to-peer network can be considered as a combination of multiple unstructured peer-to-peer networks, each of which is composed of the peers within the same group. The topology within one group is arbitrary as long as it is connected. The only constraint is on the edges between different groups. It is required that each peer is connected to at least $\mathrm{k}-1$ peers in $\mathrm{k}-1$ different groups respectively. Here $\mathrm{k}$ is multicast capacity of network. When more than k-1 peers are connected, the system reliability can be improved significantly.

The pseudo-code for peer joining is listed as Table 1 shows.

\subsection{Peer Leaving}

There are two types of peer leaving

\section{Friendly}

2. Abruptly(terminating or changing suddenly)

For the friendly leaving, the leaving peer will initiate a leaving process by sending LEAVE messages to both of its intra- neighbors and inter-neighbors. So that the system is aware of its leaving and can make necessary updates accordingly. For the abruptly leaving, the leaving peer will initiate a leaving process do not send any notification messages to both of its intra-neighbors and inter-neighbors. This is mainly due to link crash or computer crash.

\subsection{Network Coding Algorithm}

Network coding can be viewed as a generalized routing scheme and can be applied to any types of routing, i.e. uncast, multicast and broadcast, to enable a more efficient data transmission. In network coding, any intermediate node is allowed to not only forward but also combine (code) data packets received from different incoming links if necessary (Ahlswede et al, 2000; Li et al, 2003). The forwarding scheme in network coding is referred to as code-and-forward (Xing et al, 2010).

There are two main streams of research in the field of network coding. One stream investigates efficient encoding-anddecoding algorithms to increase the data transmission rate while reducing the computational cost and the other emphasizes the applications of network coding.

Finite field is also known as Galois Field and usually denoted by $\mathbf{G F}\left(2^{\mathrm{n}}\right)$ which contains $2^{\mathrm{n}}$ elements, where $\mathrm{n}$ is a positive integer. Finite field has wide applications in many areas, such as coding theory, algebraic geometry and cryptography. The following briefly describes how network coding algorithm works, including the encoding approach performed at coding nodes and decoding approach performed at receivers.

Assume each data packet in a communication network contains $\mathrm{N}$ binary bits. If we interpret every $\mathrm{n}$ consecutive bits of a packet as an element in the field GF $\left(2^{n}\right)$, the packet consists of a vector of $\mathrm{N} / \mathrm{n}$ elements. In linear network coding, the outgoing packets of a coding node are linear combinations of the incoming packets. Combining the packets requires two basic operations performed over the field GF $\left(2^{\mathrm{n}}\right)$ : addition and multiplication. Addition is the bitwise Exclusive-OR operation. For multiplication, every $\mathrm{n}$ consecutive bits, $\mathrm{b}_{0}, \mathrm{~b}_{1}$, $\mathrm{b}^{\mathrm{n}-1}$ can be interpreted as the polynomial $b_{0}+b_{1 \mathrm{x}}+\ldots+b_{n-1}$ $\mathrm{x}^{n-1}$. Hence, multiplication is done by computing the product of two polynomials.

\subsubsection{Encoding}

Network coding advocates that, in multicast network where intermediate nodes perform simple linear operation on incoming packets. The pseudo-code for encoding algorithm is listed as Table 2 shows.

\section{Table 2. Encoding Algorithm}

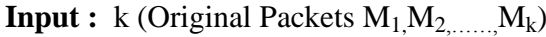
Output: $A_{\text {new }}$ (Encoded Packet)

1. Assume there are $\mathrm{K}$ original packets $\mathrm{M}_{1}, \mathrm{M}_{2, \ldots}, \mathrm{Mk}$ to be delivered from the source to one or more receivers.

2. Each packet contains encoding vector $A_{i}=$ $\left(\alpha_{1}{ }^{\mathrm{i}}, \ldots, \alpha_{\mathrm{k}}{ }^{\mathrm{i}}\right)$ and information vector $\mathrm{X}_{\mathrm{i}}=\sum_{\mathrm{k}=1}^{\mathrm{k}} \alpha_{\mathrm{k}}{ }^{\mathrm{i}} \mathrm{M}_{\mathrm{k}}$.

3. Assume there are $m$ packets $\left(\mathrm{A}_{1}, \mathrm{X1}\right), \ldots,\left(\mathrm{A}_{\mathrm{m}}, \mathrm{X}_{\mathrm{m}}\right)$ that need to be linearly coded at intermediate node.

4. The node first picks a set of coefficients $(\beta 1, \ldots$,

$$
\beta \mathrm{m}) \text { in } \operatorname{GF}\left(2^{\mathrm{n}}\right) \text {. }
$$

5. Than calculates the linear combination $\mathrm{X}_{\text {new }}=\sum^{\mathrm{m}} \mathrm{i=1}$ $\beta_{\mathrm{i}} X_{\mathrm{i}}$.

6. The new encoding vector $\mathrm{A}_{\text {new }}$ is obtained as $\mathrm{A}_{\text {new }}=$ $\left(\sum_{\mathrm{i}=1}^{\mathrm{m}} \beta_{\mathrm{i}} \alpha_{1,}^{\mathrm{i}} \sum_{\mathrm{i}=1}^{\mathrm{m}} \beta_{\mathrm{i}} \alpha_{2, \ldots \ldots,}^{\mathrm{i}} \sum_{\mathrm{i}=1}^{\mathrm{m}} \beta_{\mathrm{i}} \alpha_{\mathrm{n}}^{\mathrm{i}}\right)$.

\subsubsection{Decoding}

The receivers can than recover the original packets from the linearly combined packets, by solving a system of linear equations over a finite field. The pseudo-code for decoding algorithm is listed as Table 3 shows.

\section{Table 3. Decoding Algorithm}

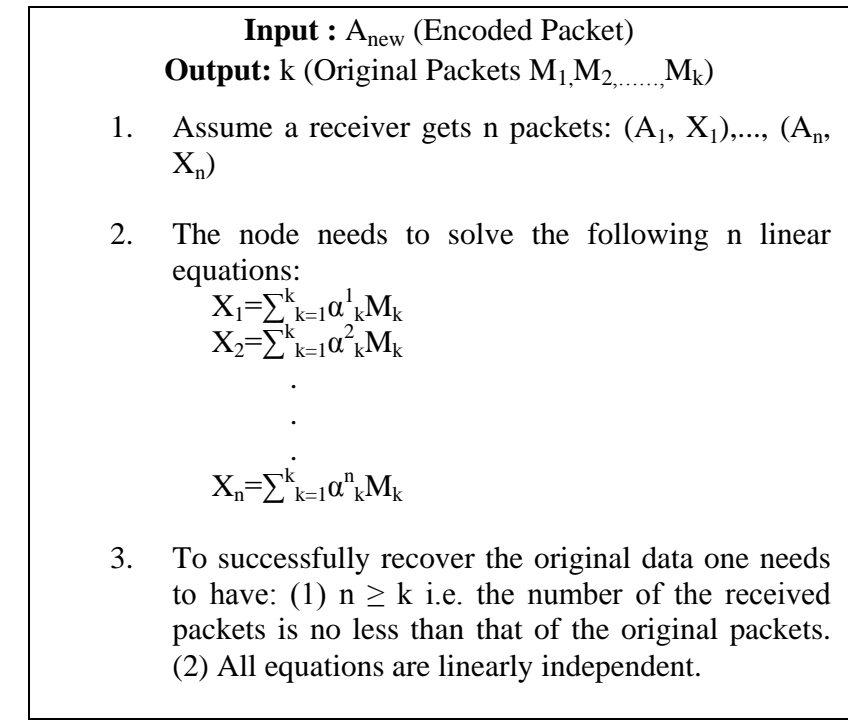

\subsection{Distributed Bellman-Ford Algorithm}

Distributed Bellman-Ford Algorithm is also known as distance vector algorithm. It is simple and quick (i.e. do not require much extra process time). Distance vector algorithm 
can be used in calculating best path (shortest path) in peer to peer network. Time complexity of distributed Bellman-Ford algorithm is $\mathrm{O}(\mathrm{VE})$ where $\mathrm{V}$ is the number of nodes and $\mathrm{E}$ is the number of link which is best complexity. Find the shortest paths, from a given source node $\mathrm{s}$ to all other nodes [9].

\section{ADVANTAGES OF PROPOSED ALGORITHM}

Applying network coding algorithms to peer to peer file sharing has some advantages.

\subsection{Throughput}

Throughput is defined as the service the peer-to-peer network provides in one time unit. Here as let different peer-to-peer network transmit the same file, thus throughput can be simply represented by the time consumed by the transmission. The shorter the time consumed, the higher the throughput. Let's start transmitting the file from time 0 . Then the consumed time is the time when the peers finish receiving the file, denoted by finish time.

\subsection{Reliability}

This performance metric is used to evaluate the ability of the peer-to-peer network to handle errors. Use the number of retransmissions to characterize this ability. A peer-to-peer network with higher reliability will have a smaller number of retransmissions, and thus higher throughput. The redundant links can greatly improve the reliability of the peer-to-peer network with little overhead.

\subsection{Link Stress}

Link stress is defined as the number of copies of the same message transmitted through the same link. It is a performance metric that only applies to an overlay network due to the mismatch between the overlay network and the physical network. Use it to evaluate the effectiveness of the topology awareness improvement and the efficiency of the peer-to-peer network.

\subsection{Scalability}

Files are distributed through a peer-to-peer network. With the increase of the network size, the total available bandwidth also increases. By using file sharing between peer-to-peer using networks coding algorithm scalability issue removes.

\subsection{Efficiency}

The network coding algorithm is deterministic and easy to implement. There is no requirement for peers to collaborate to construct the linear coding scheme on demand. All the peers need is the mapping between the group ID and the encoding function, and this mapping does not change with time. Compared to random network coding, the receiver can always recover the original messages after receiving $\mathrm{k}$ different messages and the data dissemination is more efficient as data messages are transmitted through the same overlay link at most once.

\subsection{Resilience}

Churn is a common issue in overlay networks. By adding redundant links, the negative effect of churn is eliminated.

\subsection{Heterogeneity Support}

In case that links have different link capacities, Peer-to-Peer File Sharing Based on Network Coding (PPFEED) can arrange the overlay topology to maximize the utilization of each peer's link capacity.

\section{FUTURE WORK}

Issues in network coding algorithm include code construction when two or more sources are simultaneously multicast in the network. The issue becomes more complicated when there is more than one source. For future research, the multisource multi-sink issue is a challenging issue in front of the network coding algorithms.

\section{CONCLUSION}

In network routing, messages are generally transferred by routing through intermediate nodes between the source and the destination i.e. by having intermediate nodes store and forward message. The traditional technique for multicasting in a computer network in general is not optimal. In network coding algorithm refers to as where a node is allowed to generate output messages by encoding (i.e. computing certain functions of) it's received messages. Thus, network coding allows information to mix, in contrast to the traditional routing approach where each node simply forwards received messages. Network coding algorithm can greatly improve the throughput, reliability, scalability and efficiency of a multicast network

\section{REFERENCES}

[1] Min Yang and Yuanyuan Yang," Applying network coding to peer-to-peer file sharing," IEEE TRANSACTION ON COMPUTERS, vol.63, no.8, august 2014.

[2] R. Ahlswede, N. CAI, S.-Y. R. Li, and R. W. Yeung, "Network information flow," IEEE Trans. Inf. Theory,vol. 46, no. 4,pp. 1204-1216, Jul. 2000.

[3] S.-Y. R. Li, R. W. Yeung, and N. Cai, "Linear network coding," IEEE Trans. Inf. Theory, vol. 49, no. 2, pp. 371-381, Feb. 2003.

[4] R. Koetter and M. Medard, "An algebraic approach to networkcoding,"'IEEE/ACMTrans.Netw.,vol.11,no.5,pp. 782-795,Oct.2003.

[5] T. Ho, M. Medard, J. Shi, M. Effros, and D. R. Karger, "On randomized network coding," in Proc. Annu. Allerton Conf. Commun. Control Comput. 2003, pp. 4413-4430.

[6] T. Ho, M. Medard, R. Koetter, D. Karger, M. Effros, J. Shi et al., "A random linear network coding approach to multicast," IEEE Trans.Inf. Theory, vol. 52, no. 10, pp. 4413-4430, Oct. 2006.

[7] D. S. Lun, N. Ratnakar, R. Koetter, M. Medard, E. Ahmed, and H. Lee, "Achieving minimum-cost multicast: A decentralized approach based on network coding," in Proc. IEEE INFOCOM'05 Mar. 2005, pp. $1607-1617$.

[8] D. S. Lun, M.Medard, T. Ho, and R. Koetter, "Network coding with a cost criterion," in Proc. Int. Symp. Inf. Theory Appl. (ISITA’04), Oct. 2004, pp. 1232-1237

[9] Y. Zhu, B. C. Li, and J. Guo, "Multicast with network coding in application-layer overlay networks," IEEE J. Sel. Areas Commun.,vol. 22, no. 1, pp. 107-120, Sep. 2004.

[10] Bit Torrent. (2004) [Online]. Available: http://bittorrent.com.

[11] J. W. Byers, M. Luby, and M. Mitzenmacher, "A digital fountain approach to asynchronous reliable multicast," IEEE J. Sel. Areas Commun., vol. 20, no. 3, pp. 15281540, Oct. 2002. 
[12] A. G. Dimakis, P. B. Godfrey, M. J. Wainwright, and K. Ramchandran, "Network coding for distributed storage systems," in Proc.IEEE INFOCOM'07, May. 2007, pp. 4359-4551.

[13] D. M. Chiu, R. W.Yeung, J. Huang, and B. Fan, "Can network coding help in P2P networks?" in Proc. Int. Symp. Model. Optimiz. Mobile Ad Hoc Wireless Netw. 2006, pp. 1-5.

[14] M. Kim, C. W. Ahn, M. Medard, and M. Effros, "On minimizing network coding resources: An evolutionary approach," in Proc.NetCod, 2006.

[15] K. Bhattad, N. Ratnakar, R. Koetter, and K. R.Narayanan, "Minimal network coding for multicast," in Proc. IEEE Int. Symp. Inf. Theory, Sep. 2005, pp. $1730-1734$

[16] C. K. Ngai and R. W. Yeung, "Network coding gain of combination networks," in Proc. IEEE Inf. Theory Workshop, Oct. 2004, pp. 283-287.

[17] C. Fragouli, J.Y.LeBoudec, and J.Widmer, "On the benefits of network coding for wireless applications," in Proc. Net Cod, 2006, pp. 1-6.

[18] C. Wu and B. Li, "Echelon: Peer-to-peer network diagnosis with network coding," in Proc. IEEE Int. Workshop Quality Service (IWQoS), Jun. 2006, pp. 2029.

[19] Y. H. Chu, S. G. Rao, S. Seshan, and H. Zhang, "A case for end system multicast," IEEE J. Sel. Areas Commun., Special Issue on Networking Support for Multicast, vol. 20, no. 8, pp. 1456-1471, Oct. 2002.

[20] S. Jaggi, P. Sanders, P. A. Chou, M. Effros, S. Egner, K. Jain et al., "Polynomial time algorithms for multicast network code construction," IEEE Trans. Inf. Theory, vol. 51, no. 6, pp. 1973-1982, Jun. 2005.
[21] C. Gkantsidis and P. R. Rodriguez, "Network coding for large scale content distribution," IEEE INFOCOM 2005 , Miami, FL, USA, Mar. 2005.

[22] M. Yang and Y. Yang, "An efficient hybrid peer-to-peer system for distributed data sharing," IEEE Trans. Comput., vol. 59, no. 9,pp. 1158-1171, Sep. 2010.

[23] S. Ratnasamy, M. Handley, R. Karp, and S. Shenker, "A scalable content-addressable network," in Proc. ACM SIGCOMM, 2001, pp. 149-160.

[24] (2003). Gnutella Protocol Development, the gnutella v0.6 protocol [Online]. Available: http://rfcgnutella.sourceforge.net/developer/index.html.

[25] S.Ratnasamy, M.Handley, R.M.Karp, and S.Shenker, "Topologically aware overlay construction and server selection," IEEEINFOCOM'02, New York, NY, USA, Jun. 2002.

[26] M. Yang and Y. Yang, "A hyper graph approach to linear network coding in multicast networks," IEEE Trans. Parallel Distrib. Syst., vol.21, no. 7, pp. 968-982, Jul. 2010

[27] Y. Yang, J. Wang, and M. Yang, "A service-centric multicast architecture and route protocol IEEE Trans. Parallel Distrib. Syst., vol.19, no. 1, pp. 35-51, Jan. 2008.

[28] X. Deng, Y. Yang, and S. Hong, "A flexible platform for hardware aware network experiments and a case study on wireless network coding," IEEE/ACM Trans. Netw., vol. 21 , no. 1, pp. 149-161,Feb. 2013.

[29] Y. Yang and J. Wang, "A new self-routing multicast network," IEEETrans. Parallel Distrib. Syst., vol. 10, no. 12, pp. 1299-1316, Dec. 1999.

[30] N.Cai and R.W.Yeung,"Secure network coding", Proc. IEEE International symposium on information Theory, pp.323, 2002 